\title{
Denoising, Segmentation and Characterization of Brain Tumor from Digital MR Images
}

\author{
Rahul Malhotra \\ Department of Electronics and Communication Engineering \\ BMSCE, MUKTSAR, Punjab Technical University, Jalandhar, India \\ E-mail: blessurahul@gmail.com \\ Minu Sethi (Corresponding author) \\ Department of Electronics and Communication Engineering \\ BMSCE, MUKTSAR, Punjab Technical University, Jalandhar, India \\ E-mail: minu.vohra@rediffmail.com \\ ParminderKumar Luthra \\ Department of Electronics and Communication Engineering \\ BMSCE, MUKTSAR, Punjab Technical University, Jalandhar, India \\ E-mail: pkluthra@gmail.com
}

Received: September 2, 2011

Accepted: October 8, 2011

Published: November 1, 2011

doi:10.5539/cis.v4n6p83

URL: http://dx.doi.org/10.5539/cis.v4n6p83

\begin{abstract}
The objective of this paper is to present an automated segmentation method which allows rapid identification of Tumor tissues/pathological structure with an accuracy and reproducibility comparable to those of manual segmentation. The authors uses the wiener filter for the removal of noise and then applies a new marker based watershed segmentation method using image processing and digital processing algorithms to detect Tumor tissues of Brain. This method is simple and intuitive in approach and provides higher computational efficiency along with the exact segmentation of an image. The proposed technique has been implemented on MATLAB 7.3 and the results are compared with the existing techniques.
\end{abstract}

Keywords: Brain Tumor Image, Wiener filter, Marker Based Watershed Segmentation

\section{Introduction}

A brain tumor is an abnormal growth of cells within the brain or inside the skull, which can be cancerous or non-cancerous (Marcel, Elizabeth, NathanMoon, Koen \& Guido, 2003). There are more than 120 types of brain tumors. Today, most medical institutions use the World Health Organization (WHO), classification system to identify brain tumors. The best way to determine brain tumor is to perform a type of brain scan called a Magnetic Resonance Imaging (MRI) or a scan called a Computed Tomography (CT) scan. Brain tumors are the leading cause of solid tumor death in children under age 20 now surpassing acute lymphoblast leukemia, and are the third leading cause of cancer death in young adults ages 20-39. In the states, the overall incidence of all primary brain tumors is more than 14 per 100,000 people (Matt, 2007).

In last two decades, several techniques (Health, Sarkar \& Bowyer, 1997; Zucker, 1976) have been developed by researchers to identify and locate anatomical structures from different modalities such as X-ray, CT scan and magnetic resonance imaging. A large variety of different segmentation approaches for images have been developed and published which has its own assumptions, advantages, and limitations.Health.M.D et al described that the edge detection technique works effectively on high contrast images (Health, Sarkar \& Bowyer, 1997). This method fails in detecting the edges in low contrast noisy images due to the weak gradient magnitude. Similarly, Rajani.V et al, DewalleVignin, et al, gives the algorithm of clustering based method such as K-means algorithm which have a fast speed that allows it to run on large datasets. But, its main disadvantage is that it does 
not produce same result with each run, because the resulting clusters depends on the initial random assignments (Ling Shao, 2007; Bleau \& Leon, 2000). Edge detection is a well-developed field on its own within image processing. The image is partitioned into disjoint sets (segments) by removing the edges connecting the segments. The optimal partitioning of the graph is the one that minimizes the weights of the edges that were removed (the cut). Shi's algorithm seeks to minimize the normalized cut, which is the ratio of the cut to all of the edges in the set (L.Vincent \& P.Soille, 1991; Bieniek, A. \& Moga, 2000). Region boundaries and edges are closely related, since there is oftena sharp adjustment in intensity at the region boundaries. Zhibin et al, described the fuzzy adaptive level set algorithm for brain tissue segmentation (Zhibin Chen, Tianshuang, Qiu \& Su Ruan, 2008). Marcel Prastawa et al, described the simulation of brain tumor in MR images (Marcel Prastawa, Elizabeth Bullitt, Sean Ho, \& Guido Gerig, 2009). Another basic watershed algorithm is well recognized as an efficient morphological segmentation tool which has been used in a variety of gray scale image processes \& video processing applications. However, a major problem with the watershed transformation is that it produces over segmentation. Keeping in mind the limitations of existing brain tumor segmentation methods,this paper represent an automated segmentation (CAD) tool known as Marker based Watershed algorithm to identify and segment the brain tumors in patients efficiently which requires less processing time and minimize the over segmentation problem up to a large extent (L.Vincent \& P.Soille, 1991).

\section{Proposed Marker Based Watershed Segmentation Method}

According to the definition of geography, a watershed is the ridge that divides areas drained by different river systems and catchments basins is the geographical area draining into theriver. The basic principle of watershed technique is to transform the gradient of a grey level image in a topographic surface, where the values of $f(x, y)$ are interpreted as heights and each local minima embedded in an image is referred as a catchments basins. If we imagine rain falling on the defined topographical surface, then water would be collected equally in all the catchments basins. The watershed transformation can be built up by flooding process on a gray tone image and may be illustrated by Figure 1. The basic watershed algorithm is well recognized as an efficient morphological segmentation tool which has been used in a variety of gray scale image processes \& video processing applications. However, a major problem with the watershed transformation is that it produces a large number of segmented regions in the image around each local minima embedded in theimage. Over segmentation problem in the morphological watershed segmentation for irregular-shaped objects is usually caused by spurious minima in the inverse distance transform. Figure 2 shows the over segmentation result produced by the basic watershed segmentation method. In this, different catchment basin is represented by different colors and watershed ridgelines are shown by white lines. A solution to sort out this problem is to introduce markers and flood the gradient image starting from these markers instead of regional minima .The Marker based Watershed Segmentation method possesses several important properties that makes it highly usable for various kinds of image segmentation problems. Implementation of this method involves various processing steps which can be arranged in a meaningful manner that is shown in block diagram 1.

\subsection{Raw Image Collection}

MR images of brain tumor are obtained from the NCR MRI Diagnostic Centre. The specifications of 4 image slices of patients are, Image type: T1 and T2 both with axial, coronal and sagittal view. The thickness of image slice is $0.5 \mathrm{~cm}$ and the Image format is JPEG.

\subsection{Image Pre-Processing}

In this process, the image is converted into the accessible form. Image smoothing act as the pre-processing step for image segmentation, as almost all of the images suffers from the problem of noise effect, uneven illumination and local irregularities. In this present work, the noise and uneven illumination are filtered out from the MR Images using Weiner filter which is a type of a linear filter. The contrast of the smoothened image is enhanced using the image processing toolbox functions available in Matlab 7.3. This improves the visualization effect of the original image. The imadjustfunction is used for this purpose. It has the following syntax

$$
\mathrm{J}=\text { imadjust( I, [low_in; high_in],[low_out; high_out]); }
$$

This maps the values in intensity image I to new values in J such that values betweenlow_in and high_in maps to values between low out and high out. After the intensity adjustment the image format is changed from 'indexed' to 'intensity' image into the 'binary' image. Im2bw function is used to do this process. It converts the input image to gray scale format, and then uses threshold to convert this gray scale image to binary. After this the complement of the binary image is taken to obtain the distant transform. Then the watershed function is applied on the complement of distance transform image. But watershed of negative distant transform leads to the serious over segmentation. Thus, thismethod is not preferred for the segmentation of brain MR images. So, after filtering 
and contrast enhancement, the image is first converted into double format to make it suitable for computing the gradient magnitude. The double format image is used as an input for the segmentation process.

\subsection{Proposed Marker Based Watershed Segmentation Method}

Matlab programming is used to develop algorithm for marker controlled Watershed segmentation. The gradient magnitude is used often to preprocess (Fu, K.S. \&Mui, J.K, 1981) a gray-scale image prior to using the watershed transform for segmentation which is computed using the linear filtering method. For any gray scale image $(\mathrm{x}, \mathrm{y})$, at co-ordinates $(\mathrm{x}, \mathrm{y})$, the gradient vector magnitude and angle at which maximum rate of change of intensity level occurs at the specified co-ordinates (x,y) can be computed using the equation (1) and equation (2).

$$
\begin{aligned}
& \mathrm{g}(\mathrm{x}, \mathrm{y})=\sqrt{ }\left(\mathrm{g} 1^{2}(\mathrm{x}, \mathrm{y})+\mathrm{g} 2^{2}(\mathrm{x}, \mathrm{y})\right) \\
& \alpha(\mathrm{x}, \mathrm{y})=\tan ^{-1}(\mathrm{~g} 2(\mathrm{x}, \mathrm{y}) / \mathrm{g} 1(\mathrm{x}, \mathrm{y}))
\end{aligned}
$$

Where $\mathrm{g} 1(\mathrm{x}, \mathrm{y})$ and $\mathrm{g} 2(\mathrm{x}, \mathrm{y})$ are the gradients in the $\mathrm{x}$ and $\mathrm{y}$ directions. Magnitude of these gradients is computed using the sobel mask $\mathrm{H} 1$ and $\mathrm{H} 2$, which are defined by equation (3) given below:

$$
H_{1}=\left[\begin{array}{lll}
-1 & 0 & 1 \\
-2 & 0 & 2 \\
-1 & 0 & 1
\end{array}\right], H_{2}=\left[\begin{array}{ccc}
-1 & -2 & -1 \\
0 & 0 & 0 \\
1 & 2 & 1
\end{array}\right]
$$

Watershed of simple gradient image does not produce good segmentation result (L.Vincent\&P.Soille, 1991). There are too many watershed ridge lines that do not correspond to the object in which we are interested. To overcome such a difficulty, a new approach based on the concept of markers is introduced in this project and this approach is called Marker-Controlled Watershed Segmentation.

In the present method, the internal markers are produced from the gray scale image and then external markers are found by finding pixels that are exactly midway between the internal markers. This is done by computing the watershed transform of the distance transformed image of the internal markers. The gradient image is then modified by imposing regional minima at the location ofboth the internal and external markers. The next step involves the computation of the watershed transformation of the Marker modified gradient image to produce watershed ridge lines. Finally resulting watershed ridge lines are superimposed on the original image and produce the final segmentation shown in Figure 4 and then the output image produced as a result of Region of Interest (ROI) selection is converted into label matrix. All the parameters of an object are then extracted using the image processing toolbox functions.

\section{Implementation}

The proposed algorithm is tested on Personal computer (i3 processor, 2.1GHZ,320GB HDD, 2GB RAM) using the software Matlab 7.3 with four image slices of thickness $0.5 \mathrm{~cm}$ in JPEG Format. These test images are executed in less than 13 seconds including pre-processing time on a personal computer. It also removes the problem of over segmentation in the process of watershed of negative distant transform.

\section{Results}

The final segmentation result obtained by proposed Marker based Watershed algorithm is shown in Figure 3(a) and Figure 3(b) shows the ROI of a complete tumor. Similarly, the second, third and fourth image Segmentation is shown in Figure 4, Figure 5, Figure 6.The results of the proposed algorithm for four test images are summarized in Table 1. Deviation between experimental data and data produced by the proposed method is also presented in Table 1.The values of $\mathrm{K}$ (height threshold) are optimized for proper segmentation of different images representing different tumor size and locations. And optimized values of $\mathrm{K}$ for four test images are shown in Table 1. The execution time of the algorithm for all test images are also tabulated in Table 1.

\section{Conclusions}

The developed algorithm is used to know about the location and size of the tumor. This method uses the user defined input parameters such as threshold for the analytical calculations. The optimal value of the threshold is highly dependent on shape and location of the tumor as well as on the different views of the images. In this method four images, each from different data sets are processed using the marker controlled algorithm. The result shown in Table 1 which illustrates that the developed algorithm is 8-10 times faster than current state of art in medical image segmentation. Each test image is executed in less than 15 seconds including the pre-processing time on a personal computer. The execution time for all of test images is summarized in Table 1. The marker size can be controlled interactively by the user itself. All the parameters which are extracted using the developed 
algorithm are listed in Table 1. These parameters specify the size and other dimensions of the tumor. The result obtained shows that the proposed algorithm has a higher computational efficiency along with the good performance for image segmentation problems as compared to the conventional tool. The algorithm used in this work is marker dependent which in turn depends upon the selected value of threshold. In this work, the optimal value of threshold is selected interactively. But this algorithm can be made more efficient and fast by providing the automatic value of threshold. This makes it faster and robust. In future, this work can be extended to classify the tumors using any of the techniques such as Fuzzy Expert Systems, Neural Network System, ANFIS and Hidden Markov Model.

\section{References}

Bieniek, A. \& Moga. (2000). An Efficient Watershed Algorithm Based on Connected Components. Pattern Recog., 33(6), 907-916. http://dx.doi.org/10.1016/S0031-3203(99)00154-5

Bleau, A. \& Leon, L.J. (2000). Watershed Based Segmentation and Region merging. Computer Vision and Image Understanding, 77(3), 317-370. http://dx.doi.org/10.1006/cviu.1999.0822

Dzung L. Pham, Chenyang Xu \& Jerry L. Prince. (2000). Current Methods in Medical Image Segmentation. Annual Review of Biomedical Engineering, 2, 315-337.

Fu, K.S. \& Mui, J.K. (1981). A Survey of Image Segmentation. Pattern Recog., 13(1), 3-16. http://dx.doi.org/10.1016/0031-3203(81)90028-5

Health, M.D., Sarkar, Sonaki.T \& Bowyer, K.W. (1997). A Robust Visual Method for assessing the relative performance of Edge Detection Algorithms. IEEE Trans. Pattern Anal. Machine Intel, 19(12), 1338-1359.

L. Vincent \& P. Soille. (1991). Watersheds in Digital Spaces: An Efficient Algorithm based on Immersion Simulations. IEEE Trans. Pattern and Machine Intell., 13, 583-598. http://dx.doi.org/10.1109/34.87344

Ling Shao. (2007). Partial Volume Compensated Reconstruction of Three dimensional Mass Shapes in Images.J. Digital Imaging, 20(2), 191-195. http://dx.doi.org/10.1007/s10278-007-9005-z

Marcel Prastawa BS, Elizabeth Bullitt MD, NathanMoon MS, Koen Van Leemput \& Guido Gerig. (2003). Automatic Brain Tumor Segmentation by Subject Specific Modification of Atlas Priors. Academic Radiology, 10(12), 1341-1348. http://dx.doi.org/10.1016/S1076-6332(03)00506-3

Marcel Prastawa, Elizabeth Bullitt \& Sean Ho. (2005). A brain tumor segmentation based on Edge detection. Medical Image Analysis, 170-180.

Marcel Prastawa, Elizabeth Bullitt, Sean Ho \& Guido Gerig. (2009). Simulation of Brain Tumor in MR Images for evaluation of Segmentation Efficacy. Medical Image Analysis, 13(2), 297-31. http://dx.doi.org/10.1016/j.media.2008.11.002

Matt Edman. (2007). Segmentation Using a Region Growing Algorithm. MIDAS Journal.

Zhibin Chen, Tianshuang, Qiu \& Su Ruan. (2008). Fuzzy Adaptive Level Set Algorithm for Brain Tissue Segmentation. In: $9^{\text {th }}$ International Conference on Signal Processing, Beijing, 26-29.

Zucker S.W. (1976). Region growing: childhood and adolescence. Computer Graphics image Processing, 5, 382-399. http://dx.doi.org/10.1016/S0146-664X(76)80014-7 
Table 1. Representation of Tumor Dimensions

\begin{tabular}{|c|c|c|c|c|c|}
\hline Image & $\begin{array}{l}\text { Image } \\
\text { Second }\end{array}$ & $\begin{array}{c}\text { Image } \\
\text { First }\end{array}$ & $\begin{array}{c}\text { Tumor } \\
\text { mark } \\
\text { By interior } \\
\text { of } \\
\text { Rectangle }\end{array}$ & $\begin{array}{l}\text { Medically } \\
\text { found } \\
\text { Values of } \\
\text { interior } \\
\text { Rectangle }\end{array}$ & Deviation \\
\hline Area $(\mathrm{mm} 2)$ & 355 & 419.4 & 106 & 112.5 & $5.7 \%$ \\
\hline $\begin{array}{l}\text { Major Axis } \\
\text { Length(mm) }\end{array}$ & 21.7 & 25.8 & 11.57 & 10.9 & $5.6 \%$ \\
\hline $\begin{array}{l}\text { Minor Axis } \\
\text { Length(mm) }\end{array}$ & 21.1 & 21.2 & $10 . .92$ & 10.33 & $5.7 \%$ \\
\hline $\begin{array}{l}\text { Eccentricity } \\
0<\mathrm{e}<1\end{array}$ & 0.20 & 0.57 & 0.31 & 0.325 & 4. $6 \%$ \\
\hline $\begin{array}{l}\text { Orientation } \\
\text { In Degree }\end{array}$ & -26 & -62.4 & -24.2 & -23.1 & 4. $5 \%$ \\
\hline Equidiameter(mm) & 21.3 & 23 & 11.48 & 11.97 & 4. $1 \%$ \\
\hline $\begin{array}{l}\text { Solidity } \\
0<\mathbf{s}<1\end{array}$ & 0.98 & 0.94 & 0.98 & 0.94 & 4. $3 \%$ \\
\hline Perimeter (mm) & 70.8 & 85 & 39.93 & 42.25 & $6.0 \%$ \\
\hline Values of k(pixels) & 80 & 82 & 30 & N. A. & N. A. \\
\hline Processing Time (in seconds) & 13 & 14 & 13 & 105 & $\begin{array}{l}8 \text { times } \\
\text { faster }\end{array}$ \\
\hline
\end{tabular}




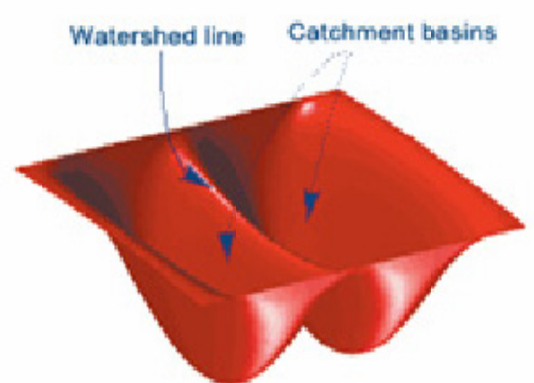

Figure 1. Watershed segmentation simplified to 2 Dimensions

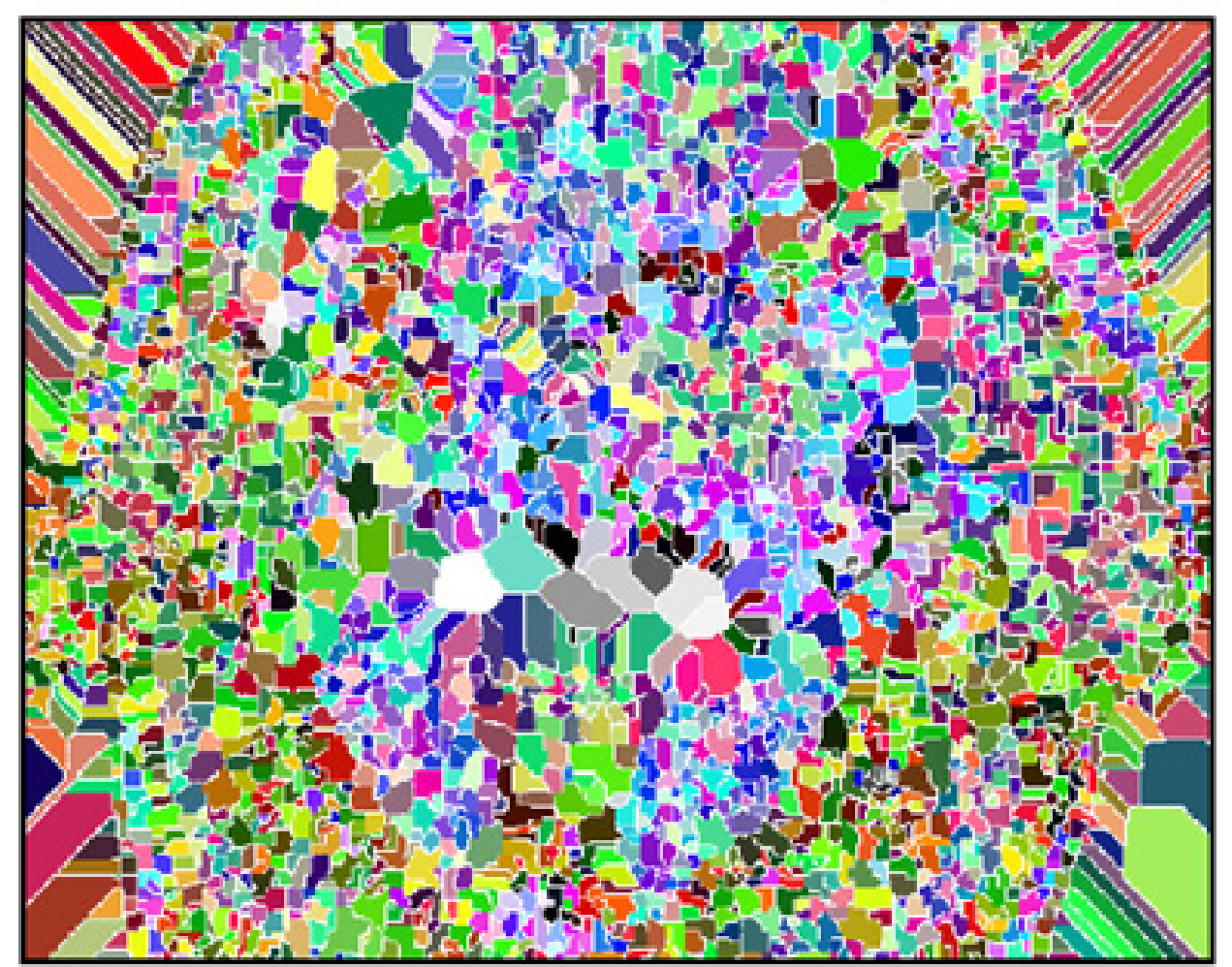

Figure 2. The over segmentation result produced by the basic watershed segmentation method 


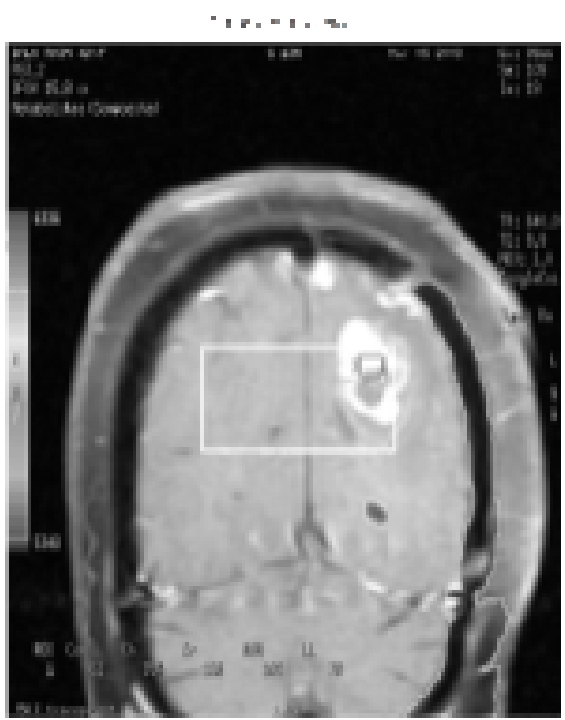

Figure 3. (a) Final segmentation results obtained after applying watershed on modified gradient image

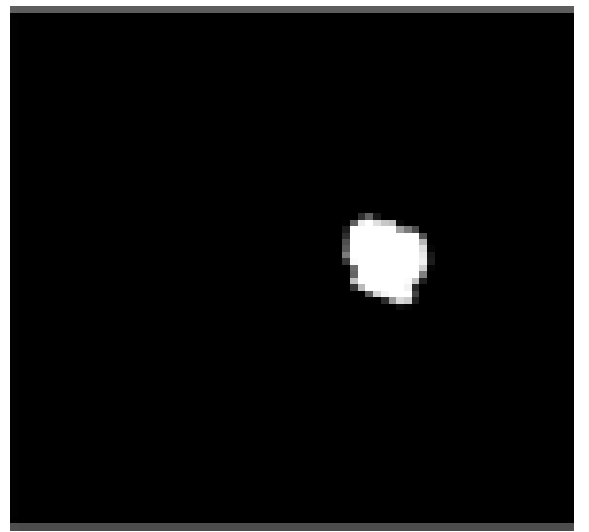

Figure 3. (b) ROI of complete tumor 
Similarly, the second image Segmentation is shown in Figure 4.

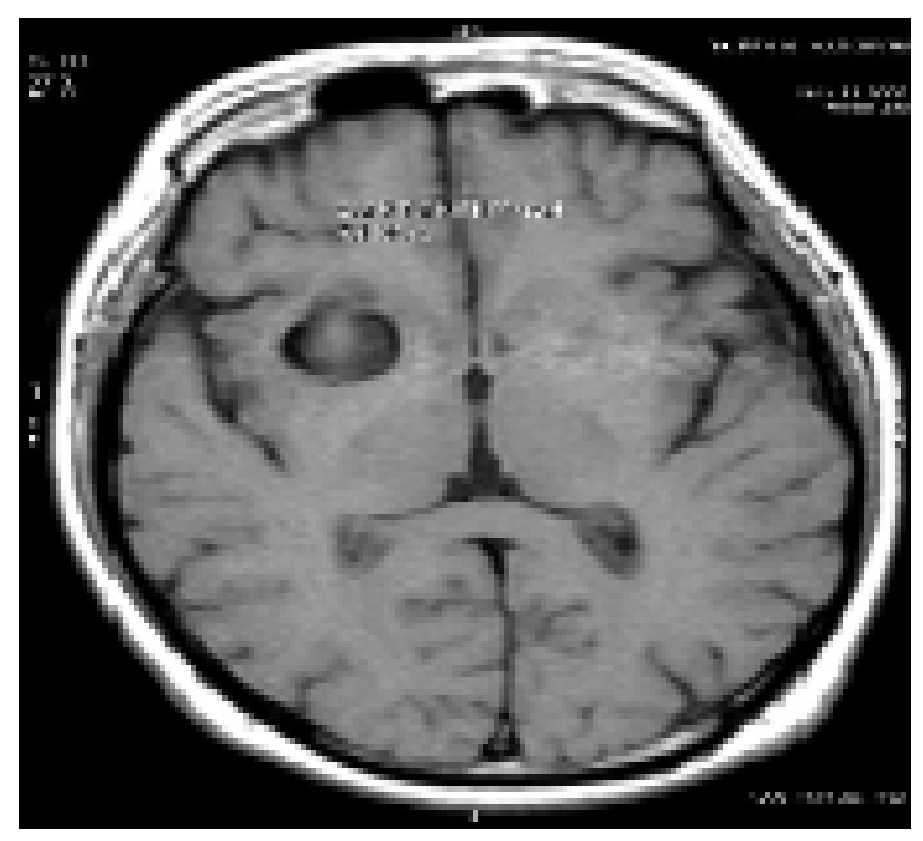

Figure 4. (a) Original image

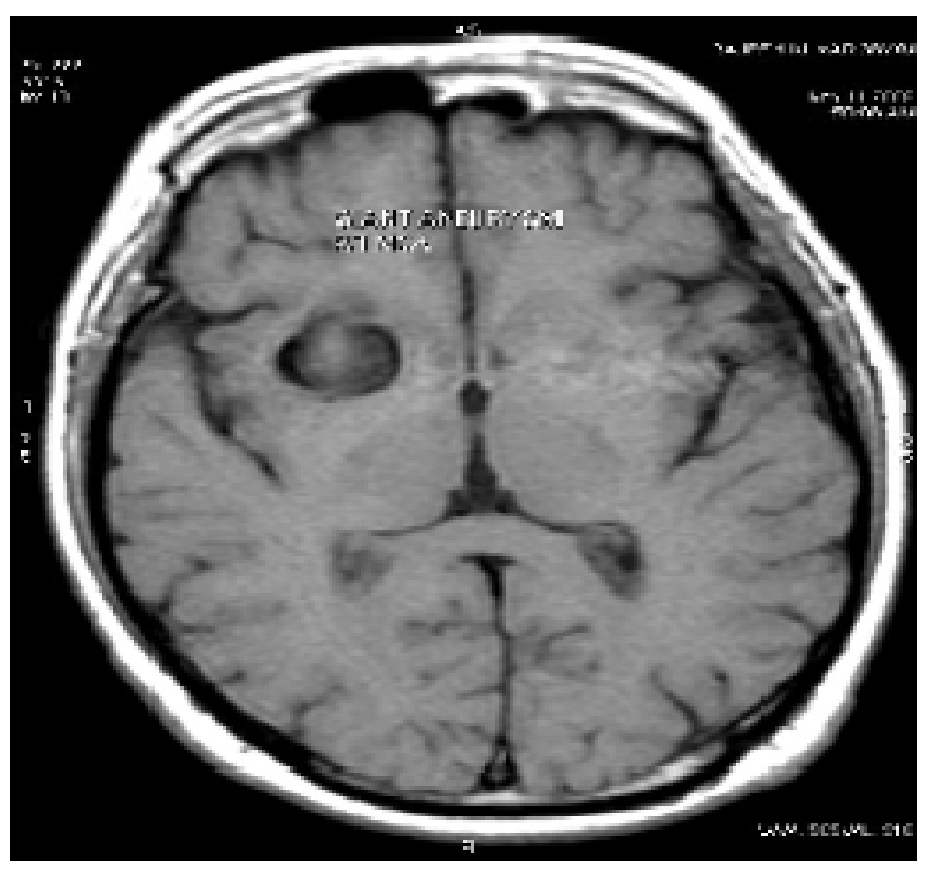

Figure 4. (b) Filtered and contrast enhanced image 


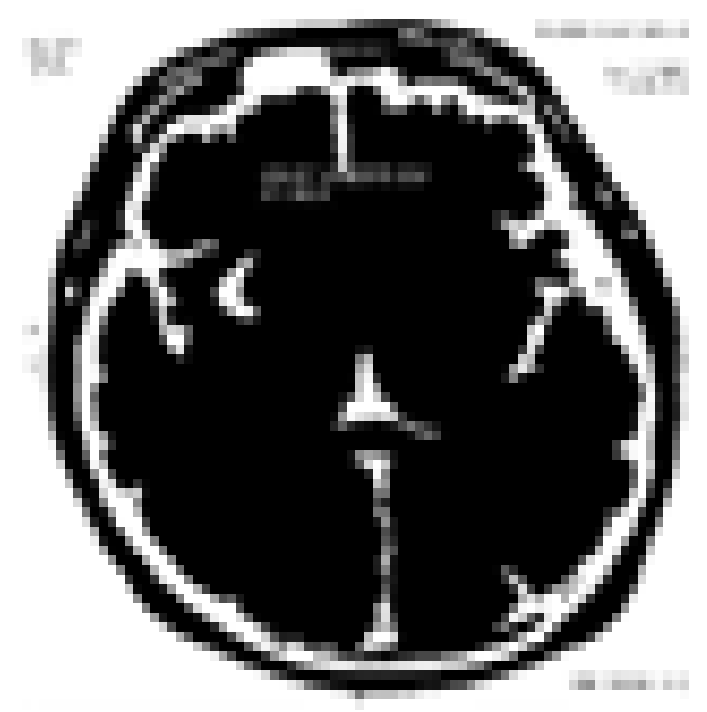

Figure 4. (c) Internal markers

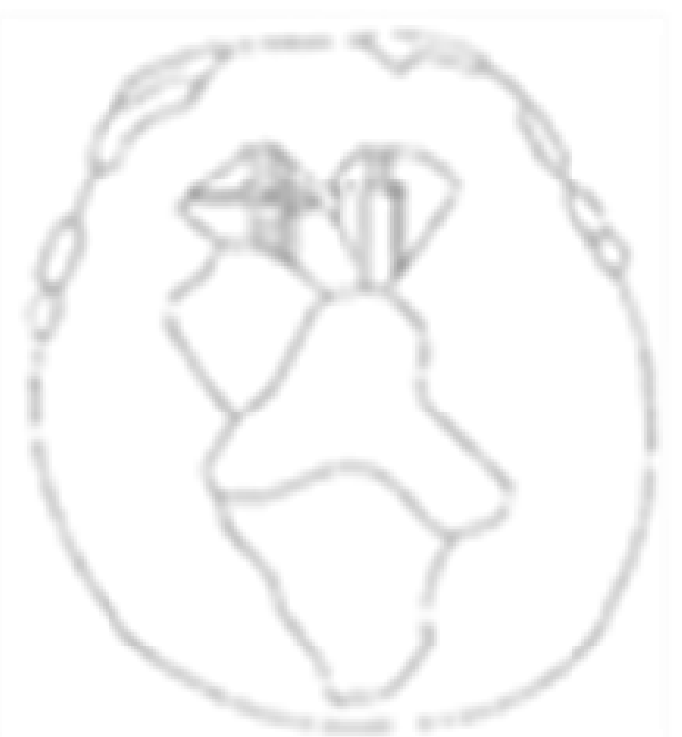

Figure 4. (d) External markers 


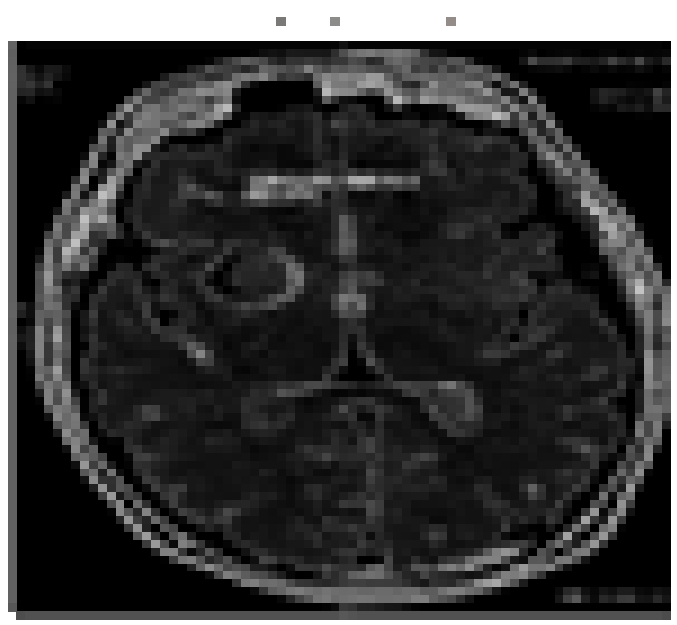

Figure 4. (e) Modified gradient image

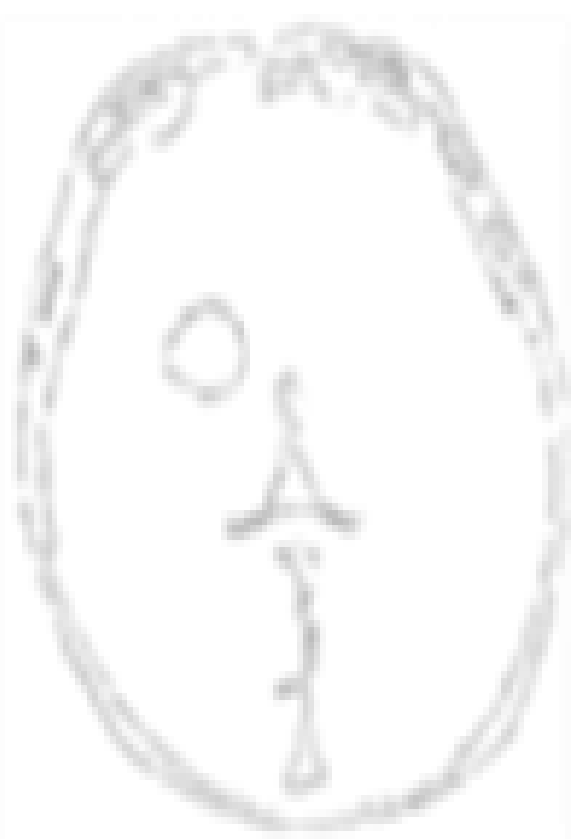

Figure 4. (f) Watershed of modified gradient image 


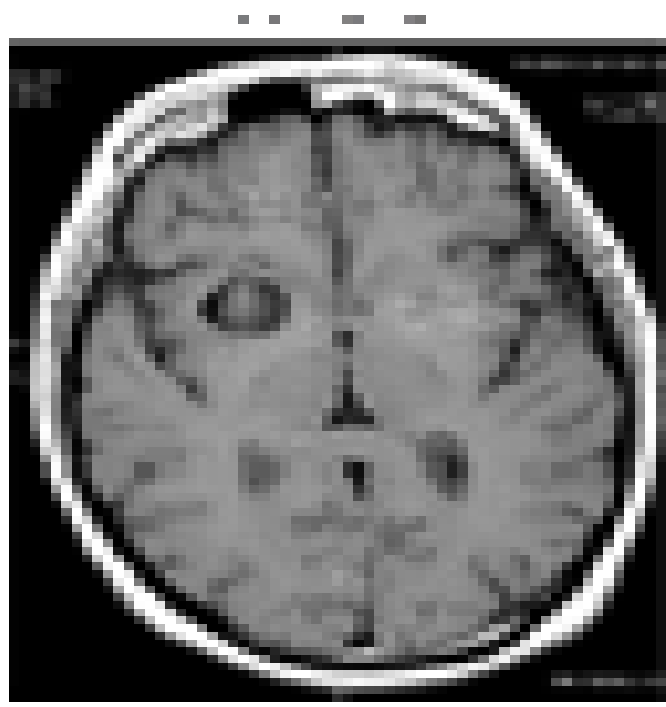

Figure 4. (g) Final segmentation

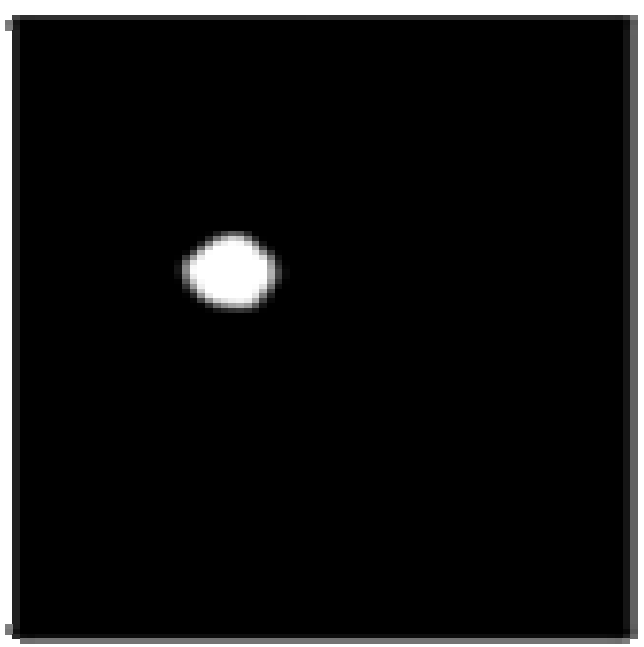

Figure 4. (h) Segmented ROI using ROI command 
The segmentation of third image (Figure 5 (a)) produces the result as shown in Figure 5 (b) to 5 (k).

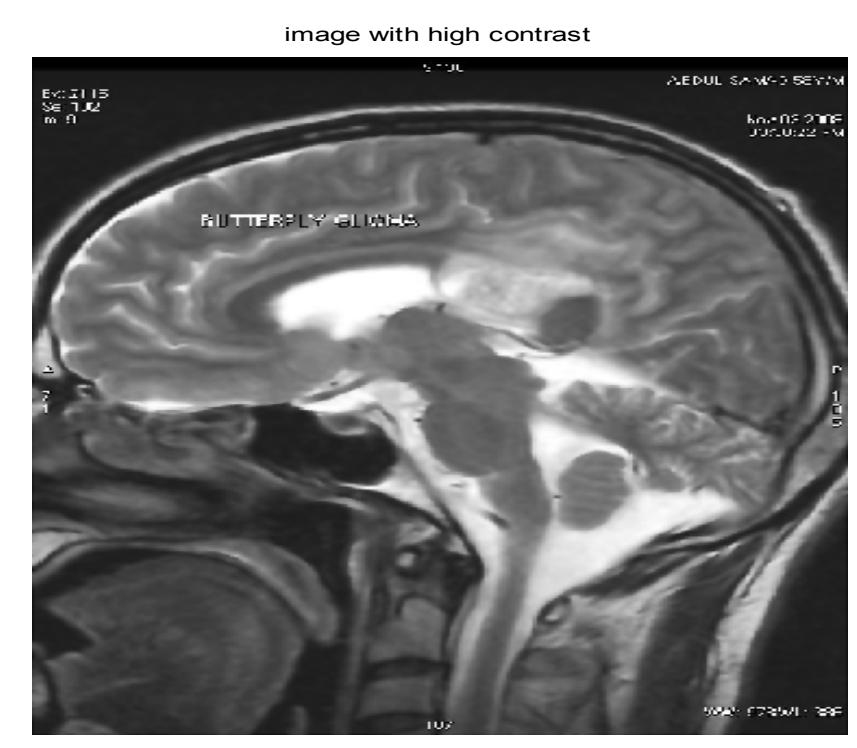

Figure 5. (a) Original sagittal image

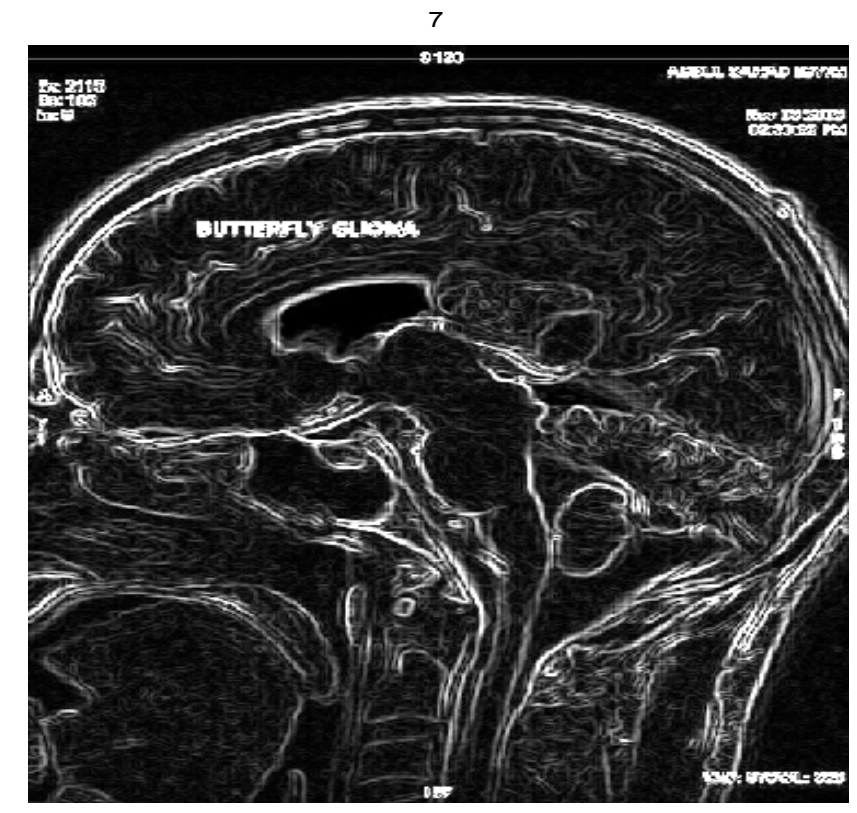

Figure 5. (b) Contrast enhancement after filtering 


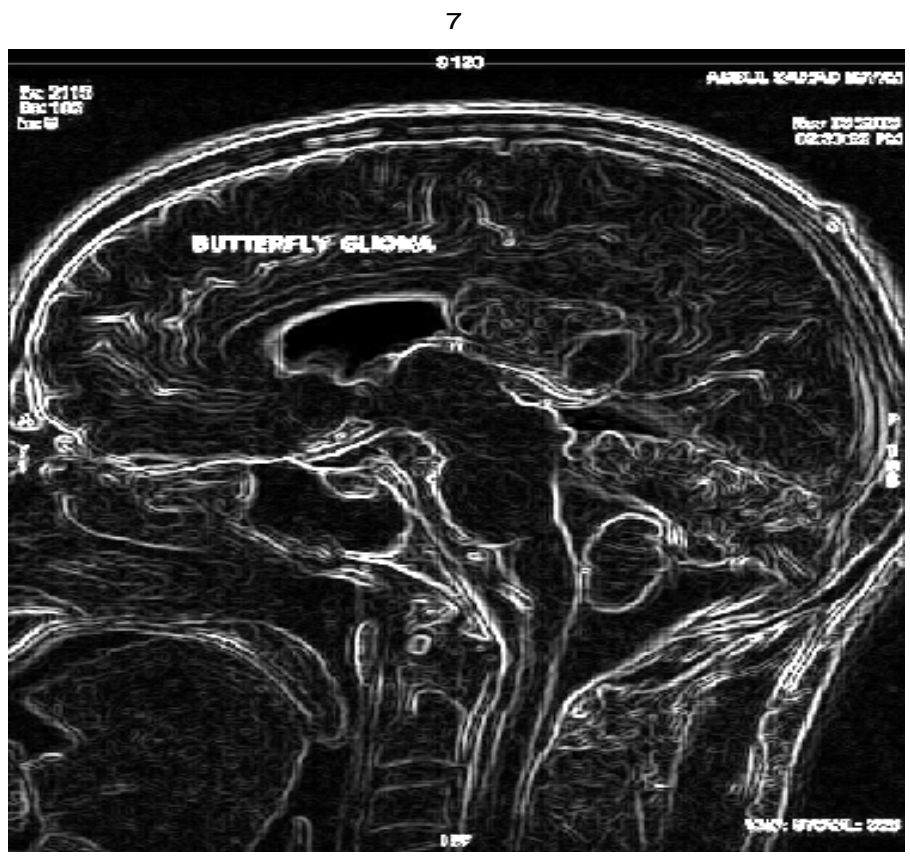

Figure 5. (c) Gradient image

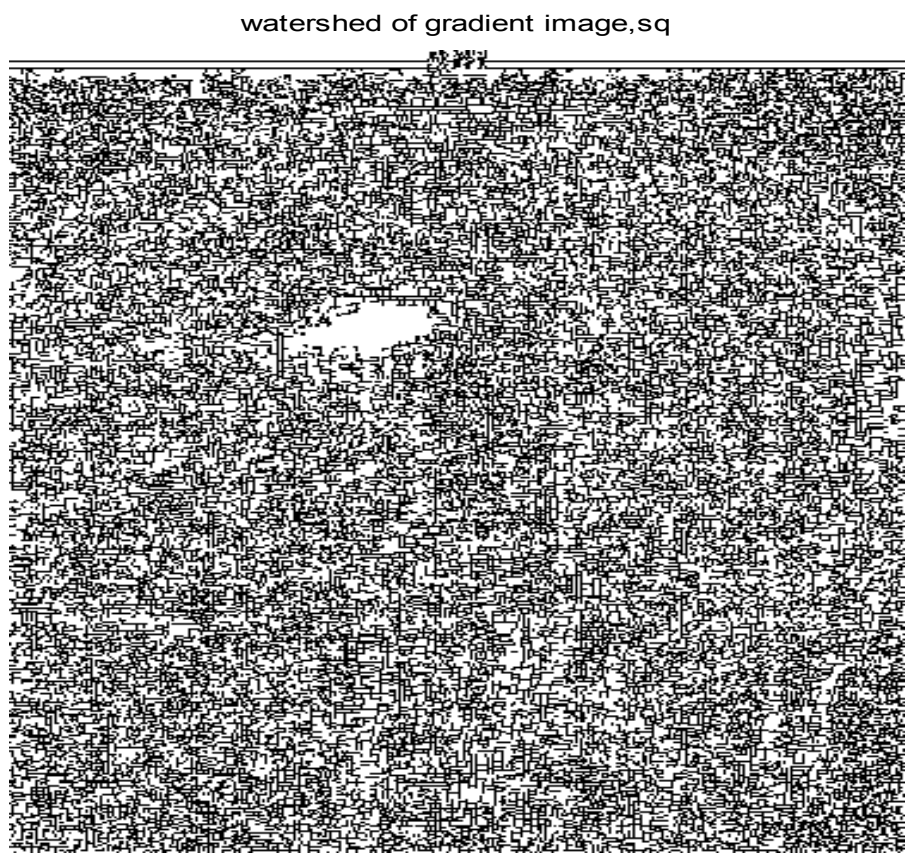

Figure 5. (d) Watershed of gradient image 


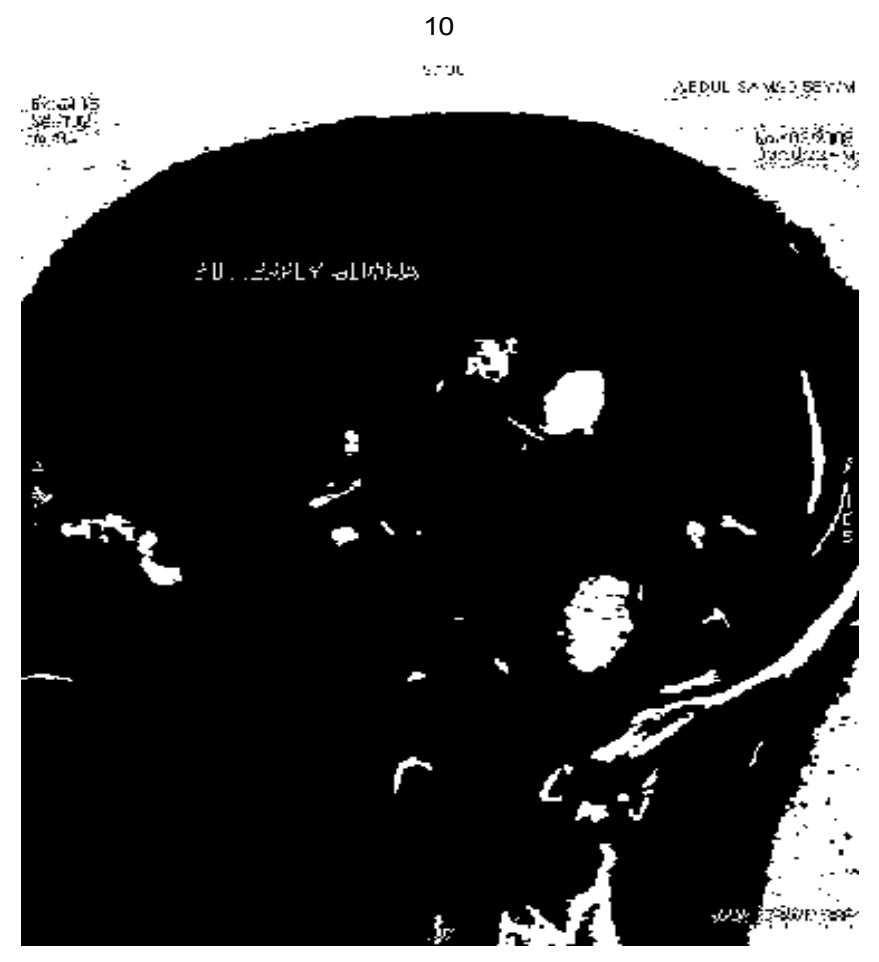

Figure 5. (e) Internal markers

$\operatorname{fim}(\mathrm{im})=255,11$

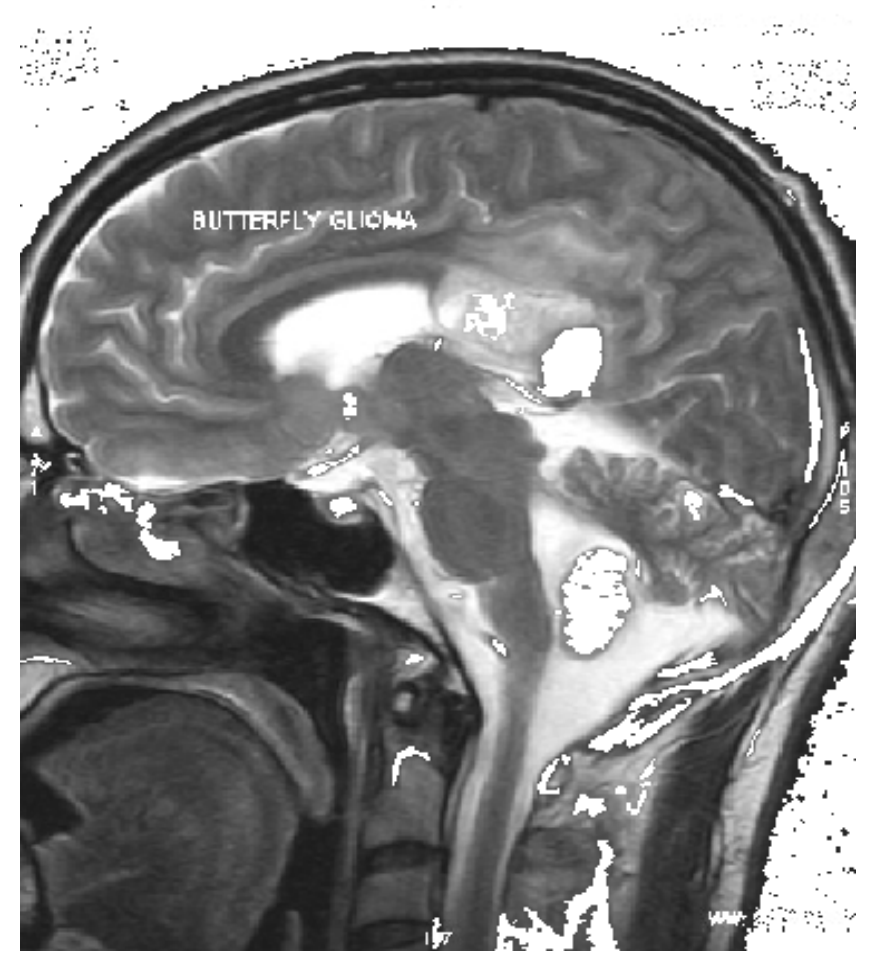

Figure 5. (f) Internal markers superimposed on original image 


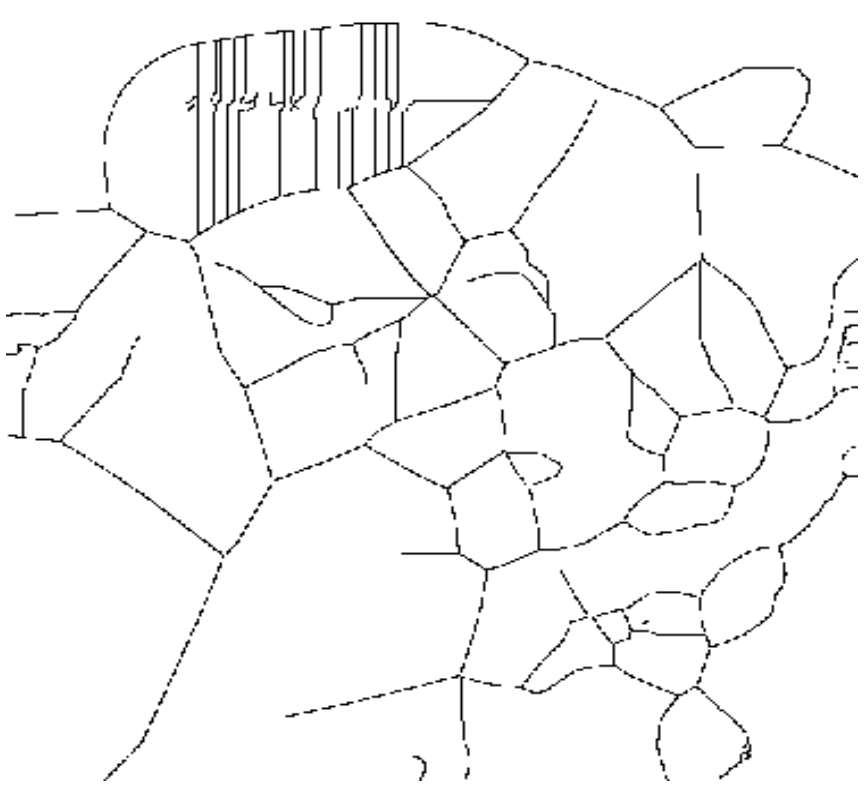

Figure 5. (g) External markers

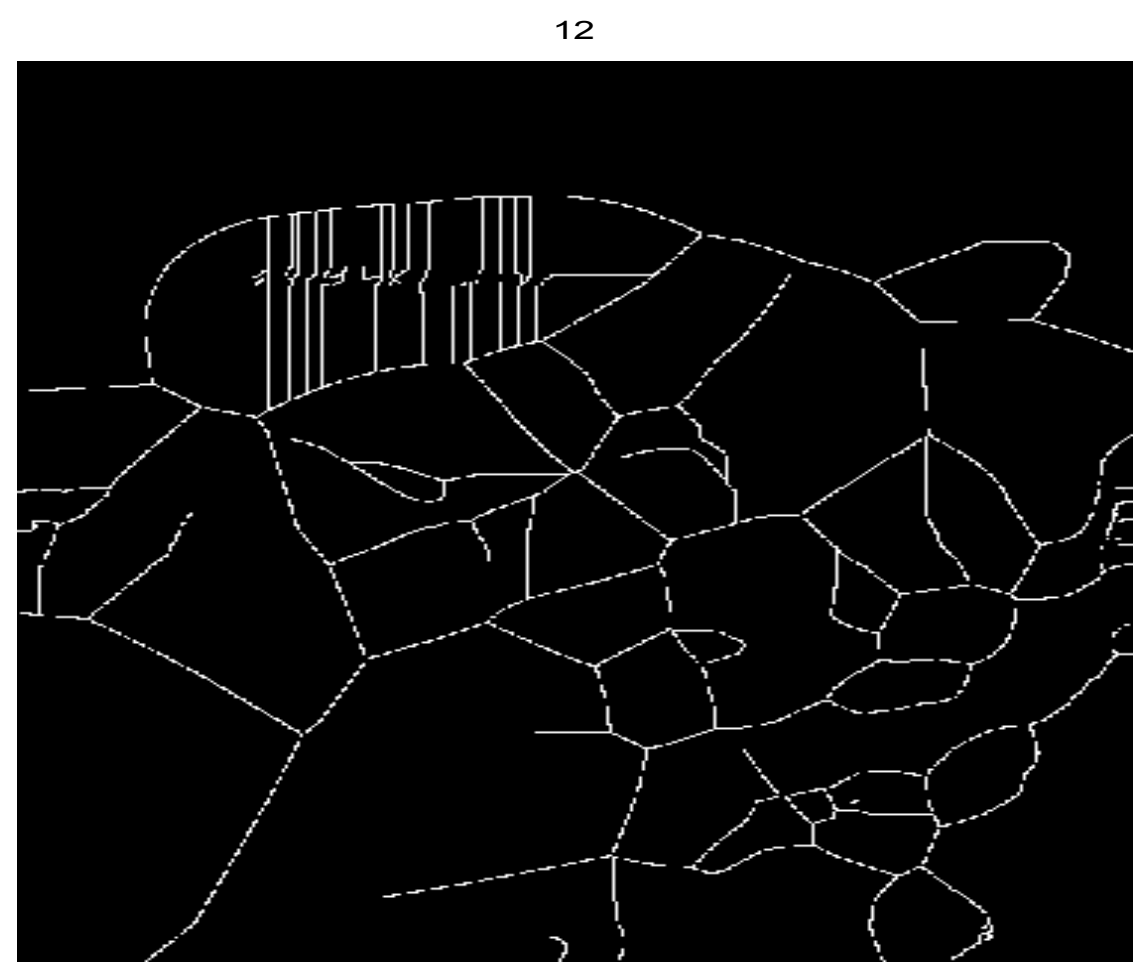

Figure 5. (h) Distance transform of external markers 


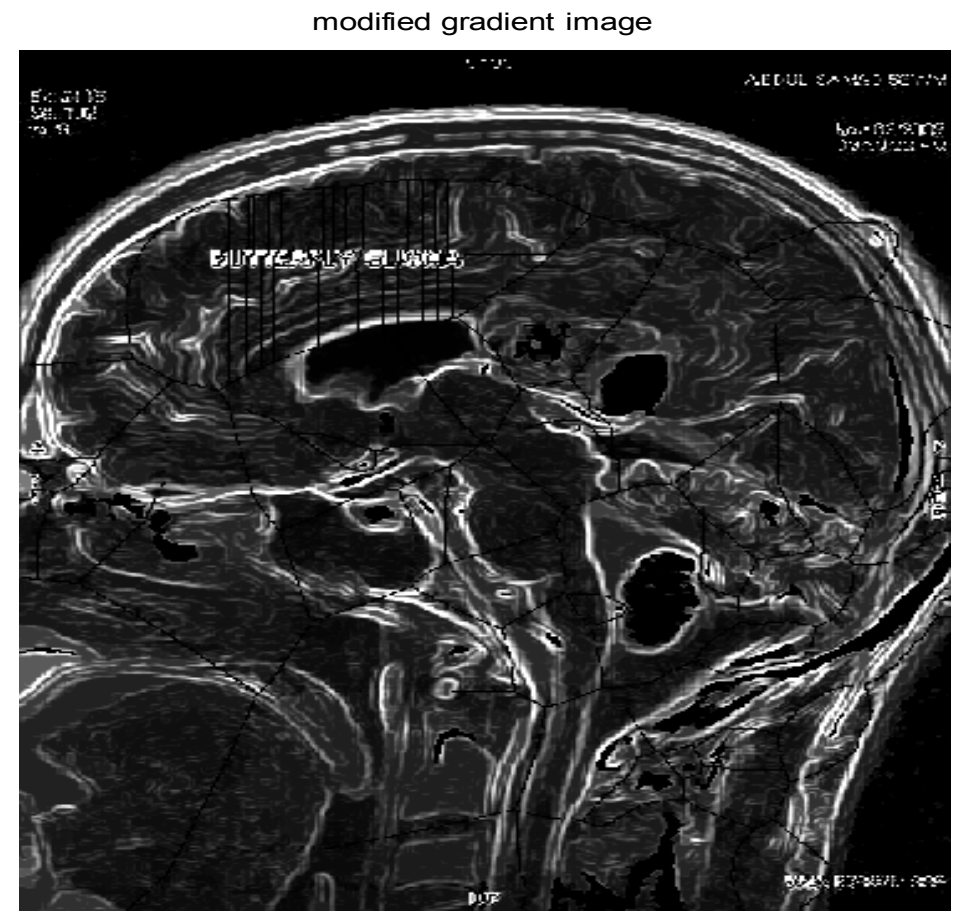

Figure 5. (i) Modified gradient image

watershed of modified gradient image

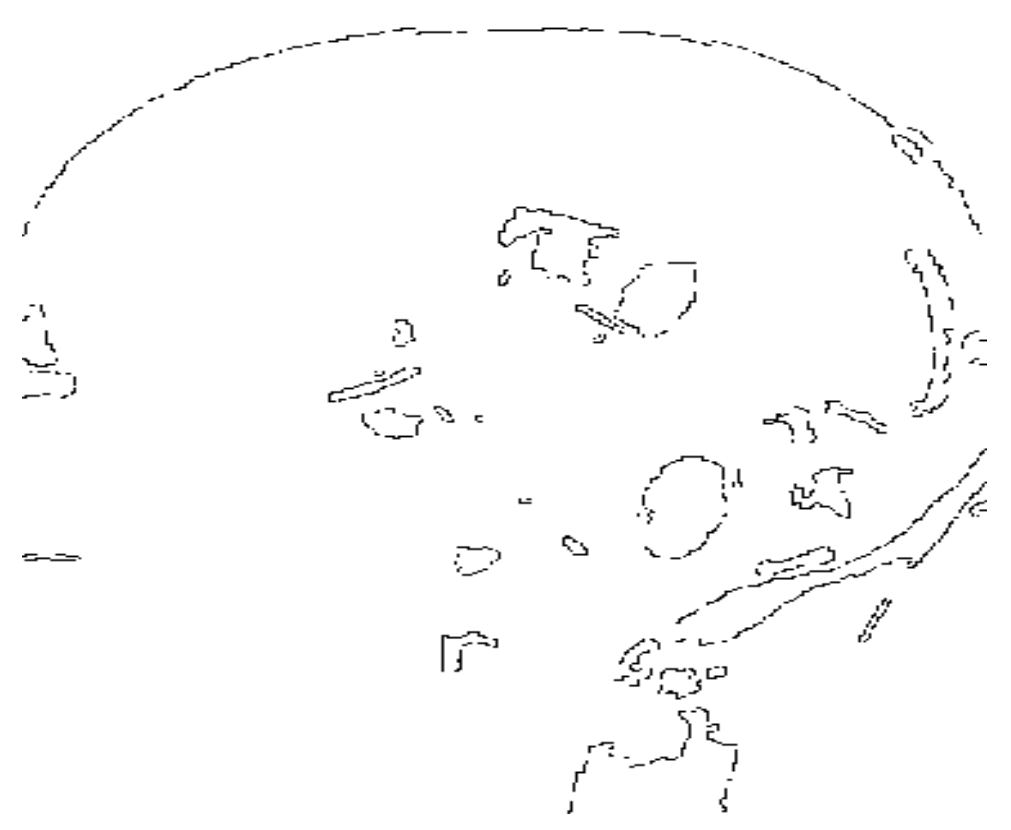

Figure 5. (j) Watershed ridgelines of mod gradient image 


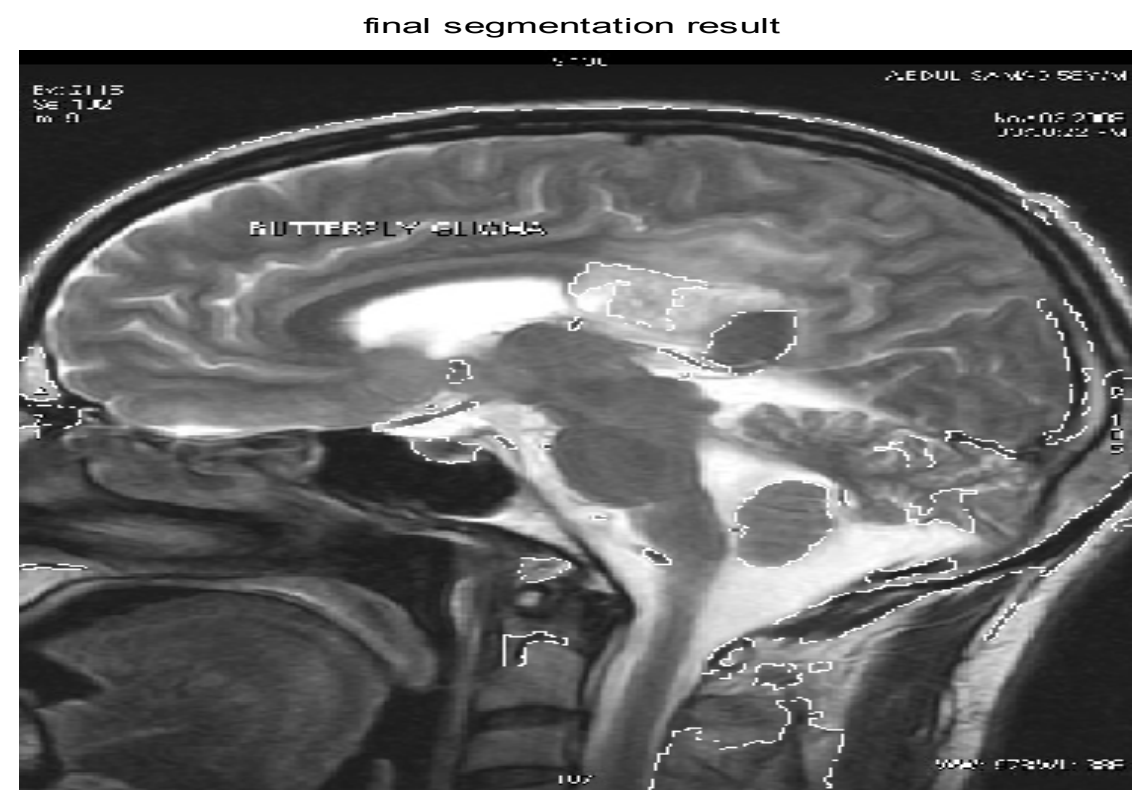

Figure 5. (k) Final segmentation

The image shown in Figure 6 is the axial view of the head. This image is also segmented in the same way as the previously shown images have been segmented.

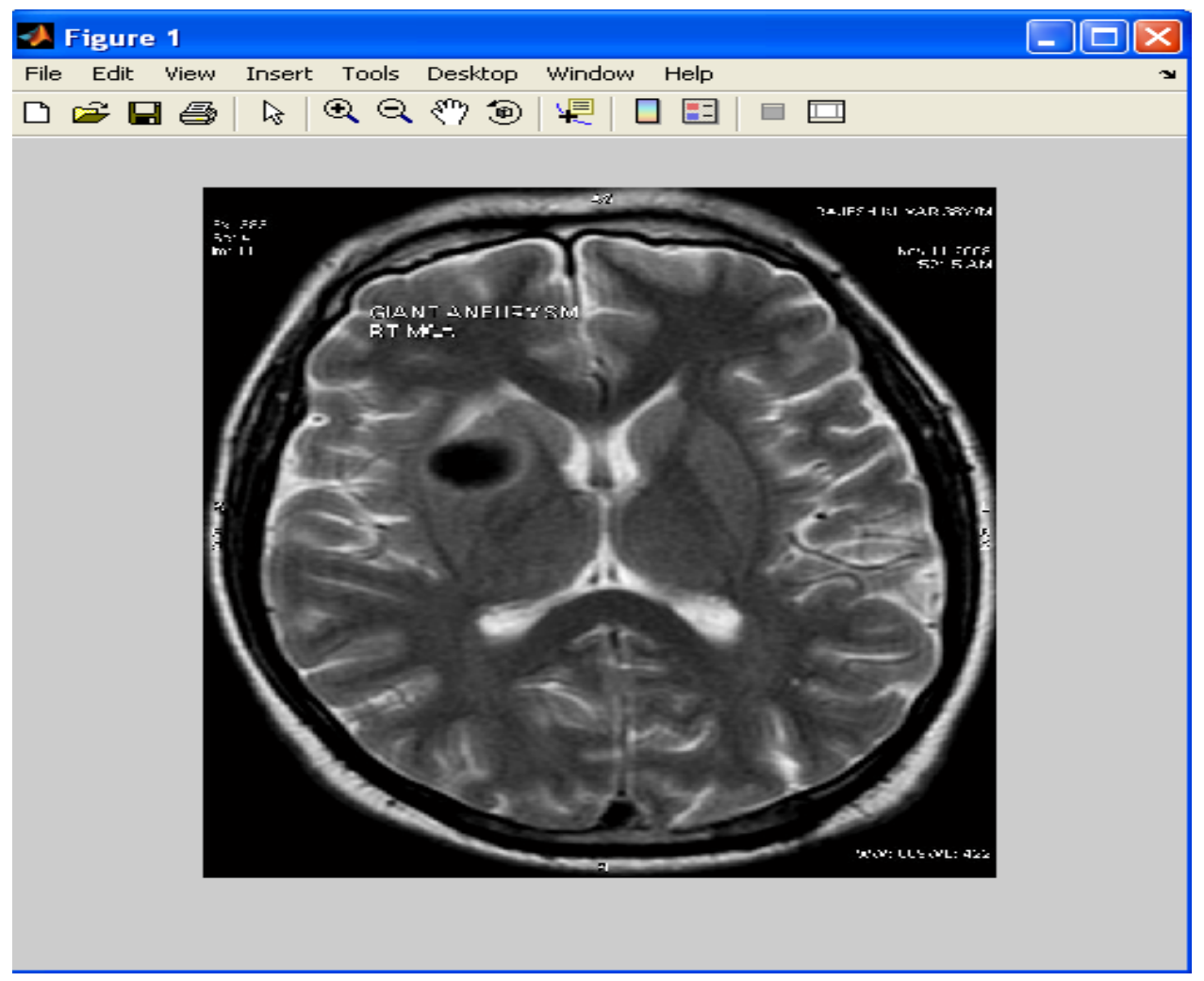

Figure 6. (a) Original sagittal image 


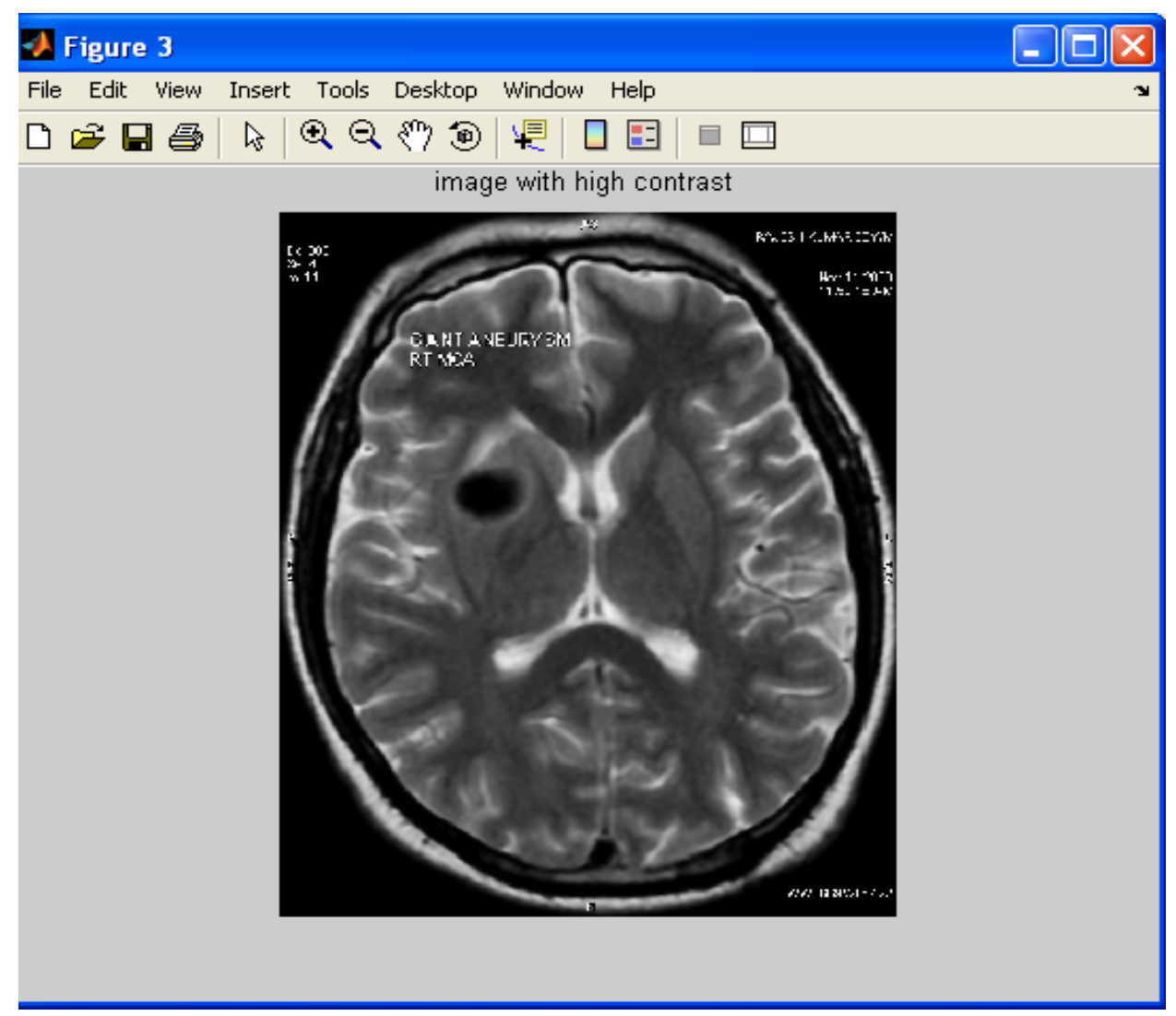

Figure 6. (b) Contrast enhancement after filtering

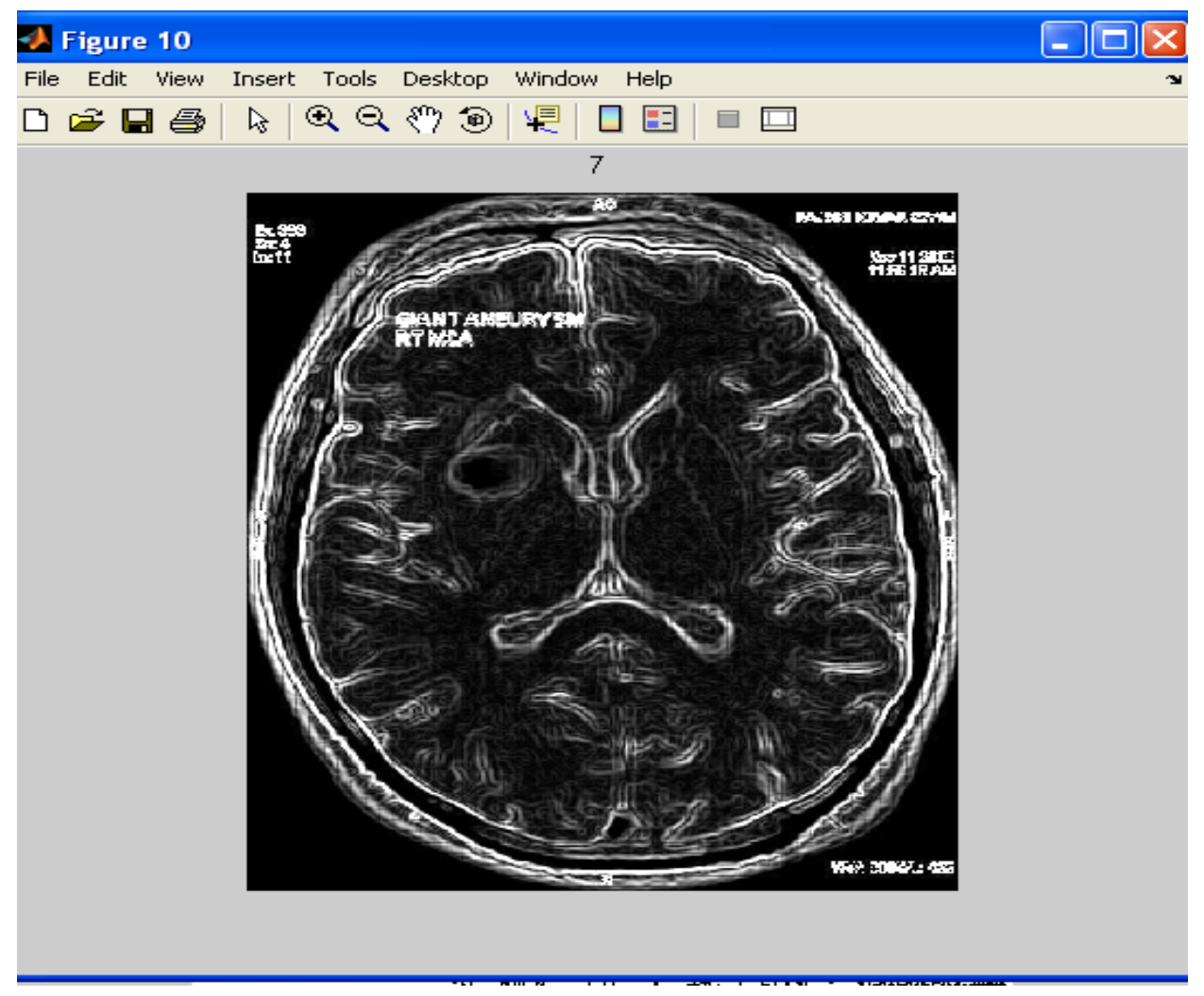

Figure 6. (c) Gradient image 


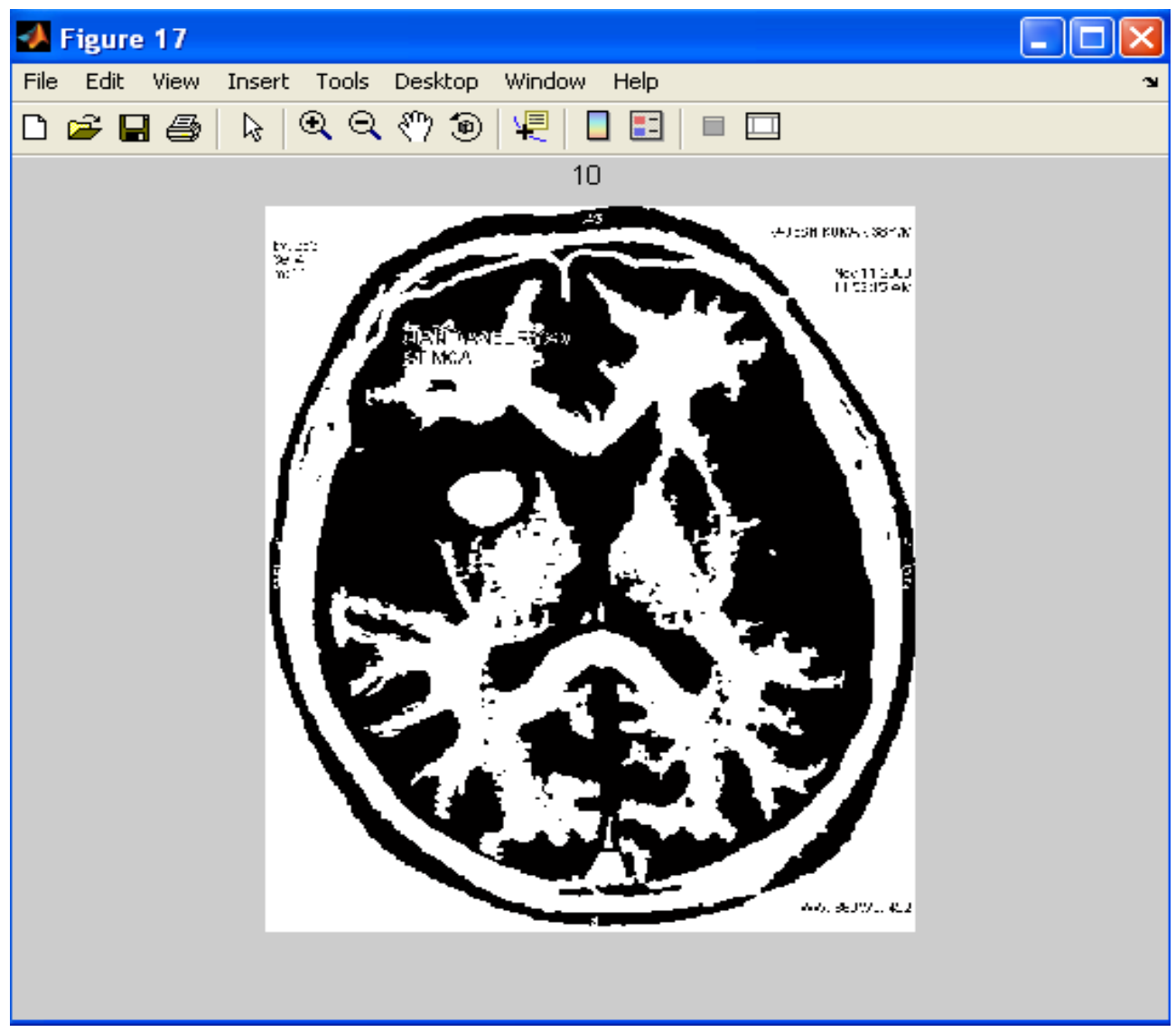

Figure 6. (d) Watershed of gradient image

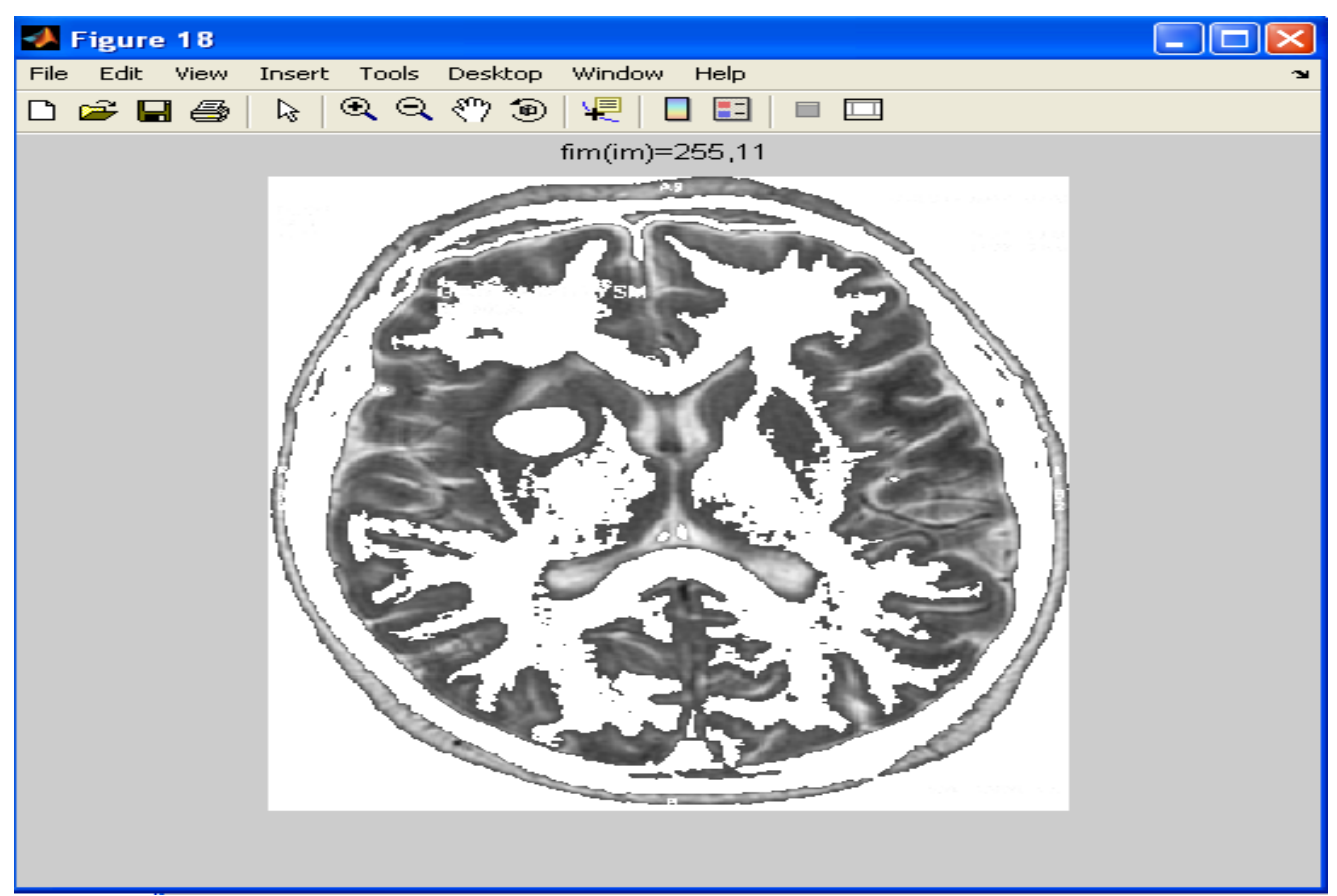

Figure 6. (e) Internal markers 


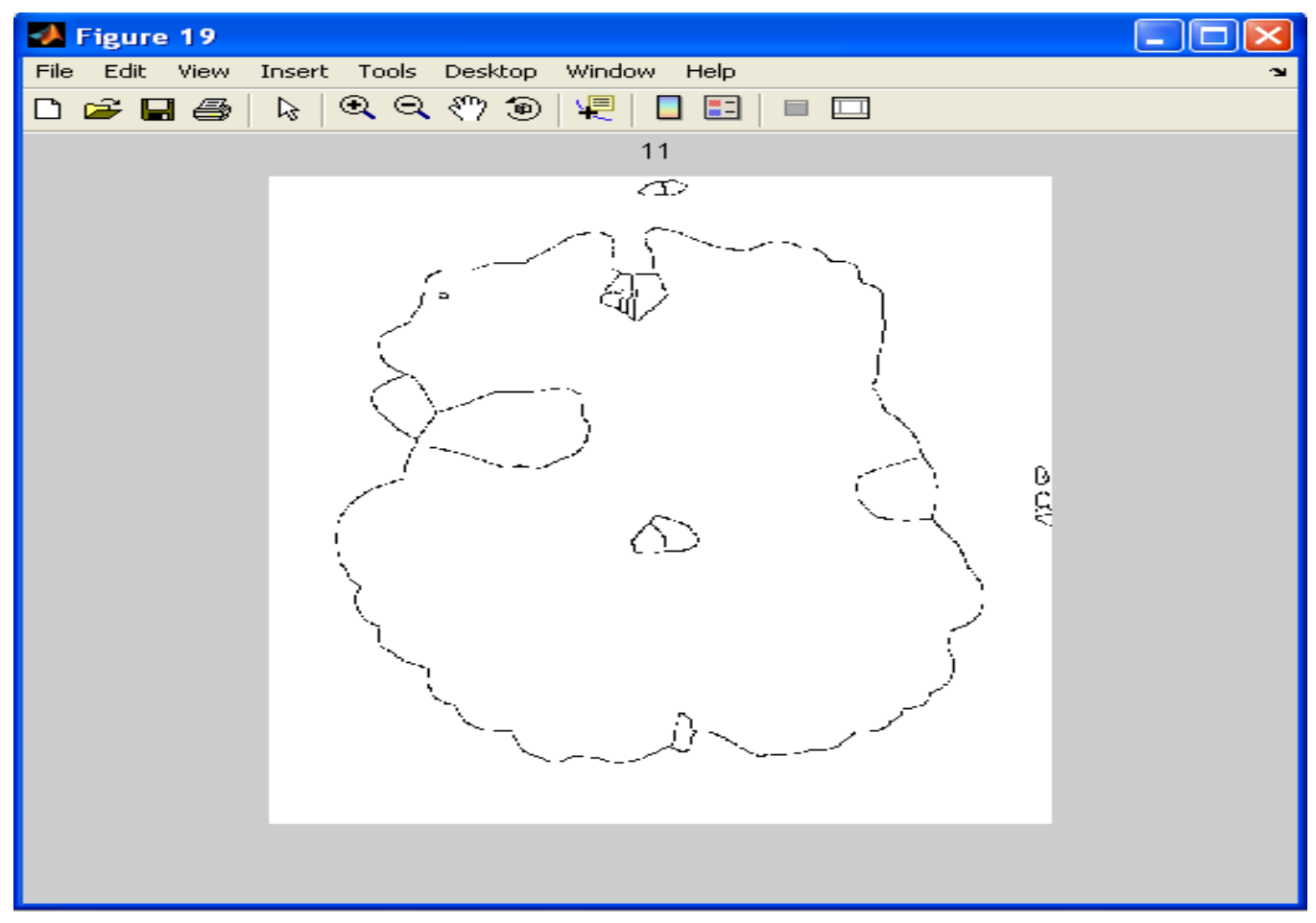

Figure 6. (f) Internal markers superimposed on original image

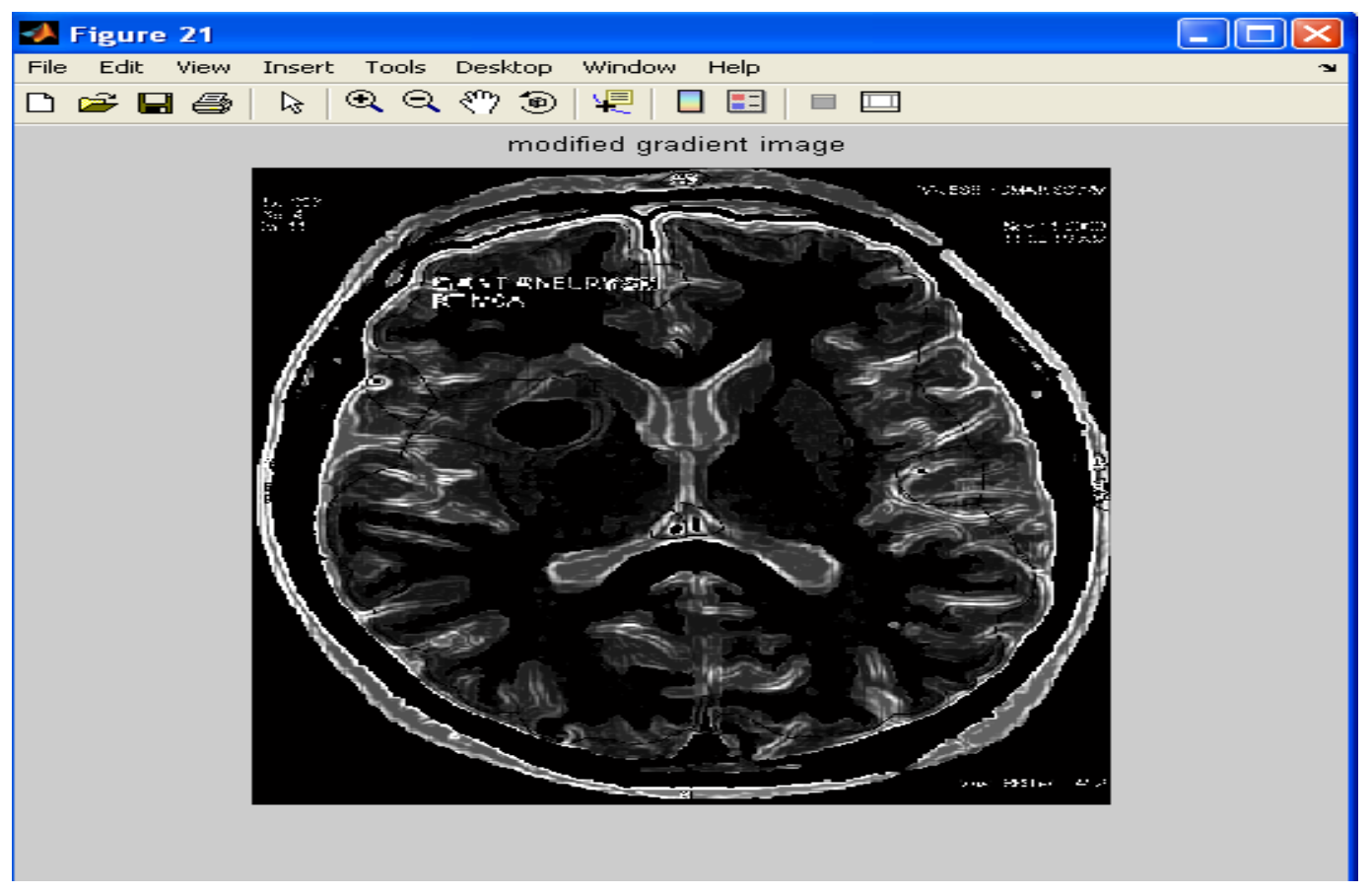

Figure 6. (g) External markers 


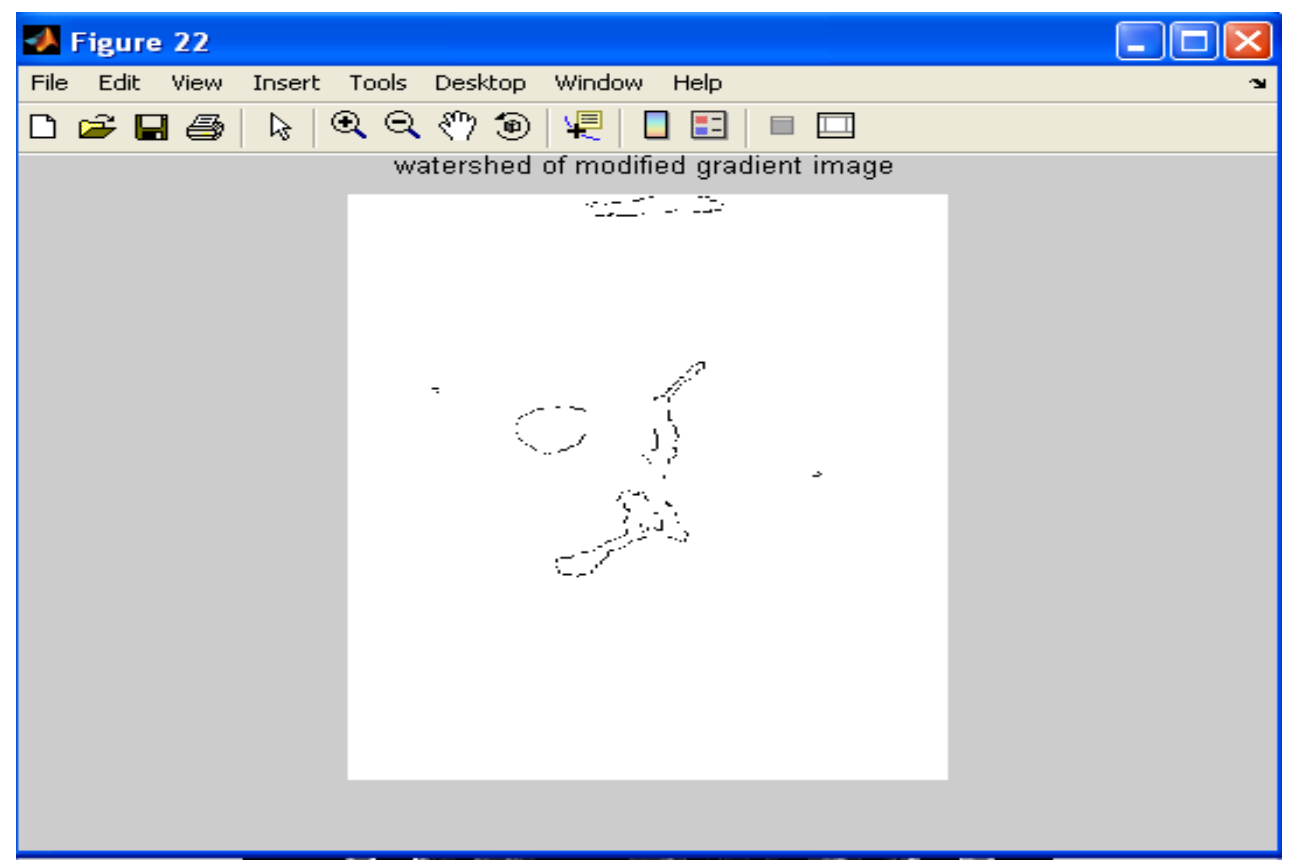

Figure 6. (h) Modified gradient image

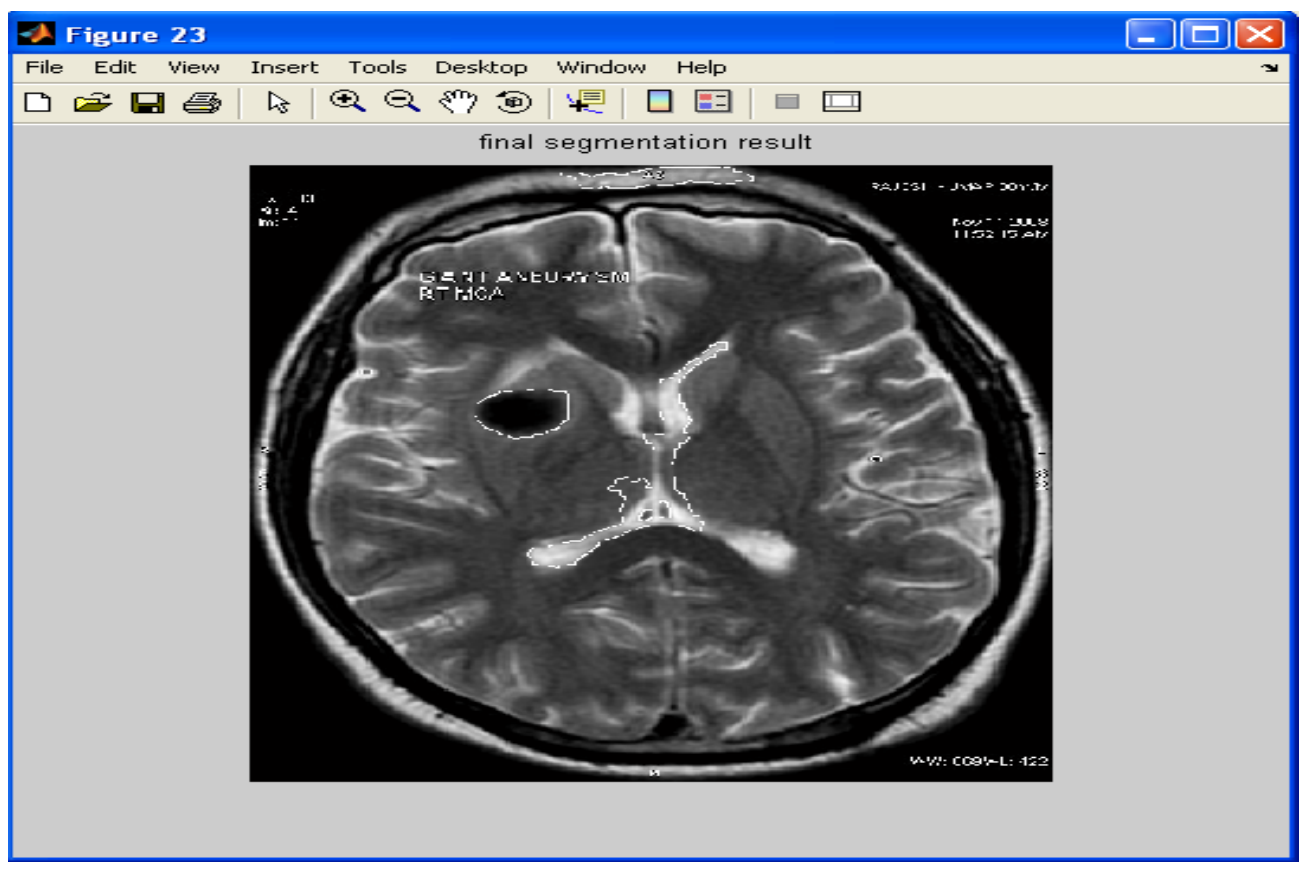

Figure 6. (i) Watershed ridgelines of mod gradient image 


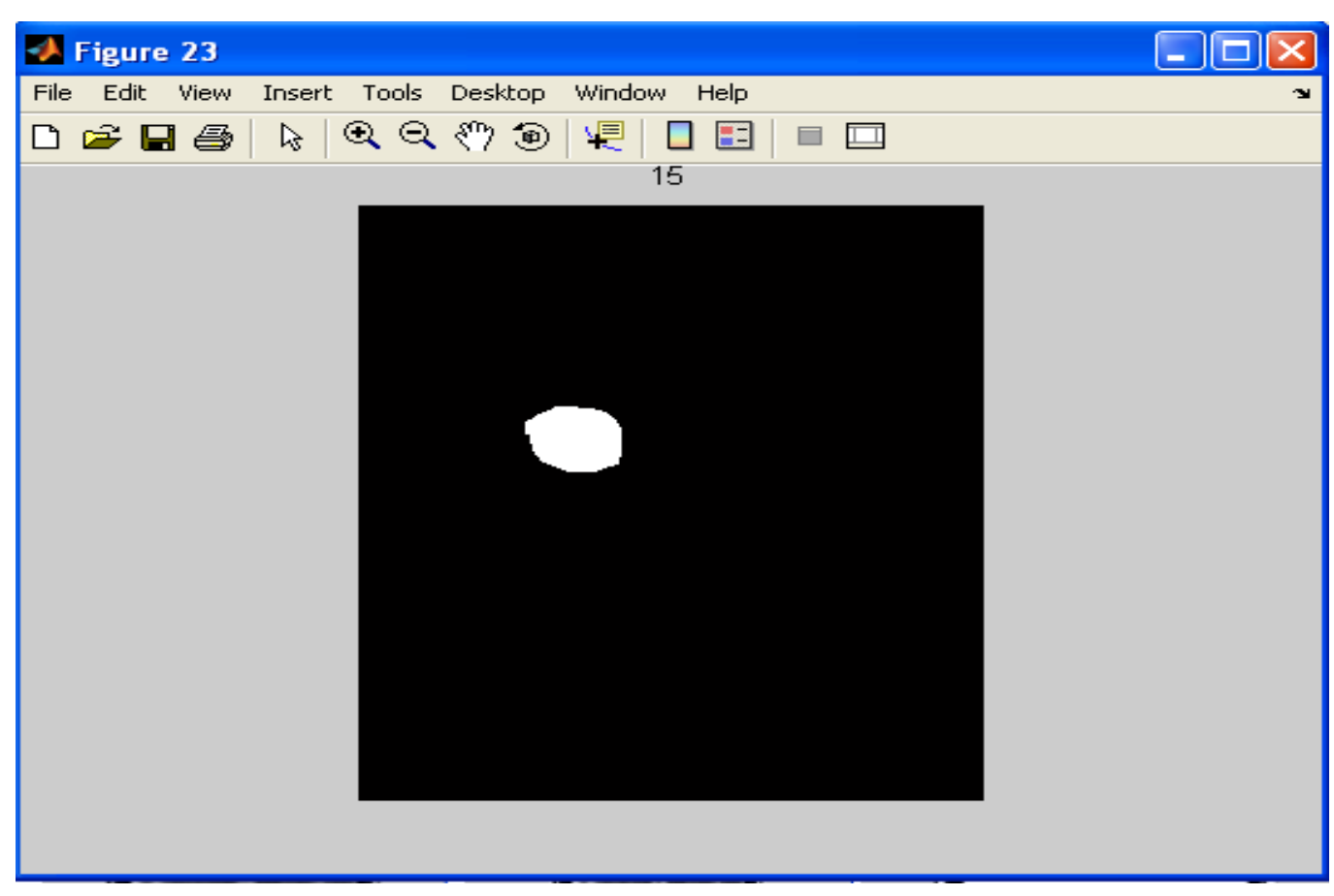

Figure 6. (j) Final segmentation

Block diagram 1. Representation of marker based watershed segmentation process

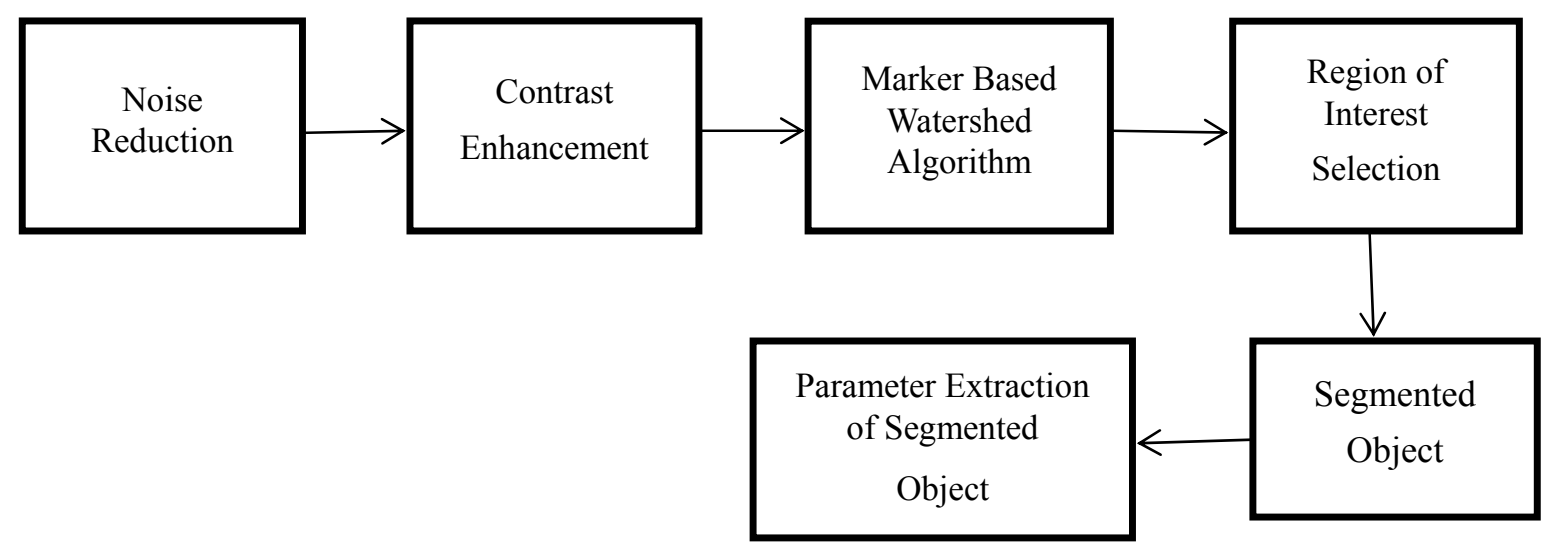

